TAMKANG JOURNAL OF MATHEMATICS

Volume 33, Number 2, Summer 2002

\title{
TWO NEW DISCRETE OU-IANG TYPE INEQUALITIES
}

\section{WEN-RONG LI AND SUI SUN CHENG}

\begin{abstract}
Two discrete Ou-Iang type inequalities are established which are supplementary to some of the existing results.
\end{abstract}

In [1], several explicit bounding sequences are found for nonnegative sequences of the form $\left\{x_{n}\right\}_{n=\alpha}^{\infty}$ which satisfy

$$
x_{n}^{p} \leq \gamma_{n}+\sum_{m=\alpha}^{n-1} f_{m} x_{m}^{p}+\sum_{m=\alpha}^{n-1} g_{m} x_{m}^{q}, n \geq \alpha,
$$

where $\alpha$ is an integer, $p, q \geq 0$ and $\left\{f_{n}\right\}_{n=\alpha}^{\infty},\left\{g_{n}\right\}_{n=\alpha}^{\infty},\left\{\gamma_{n}\right\}_{n=\alpha}^{\infty}$ are nonnegative sequences.

Here we are interested in obtaining additional explicit bounding sequences for nonnegative sequences of the form $\left\{x_{n}\right\}_{n=\alpha}^{\infty}$ which satisfy the above or the following functional relation

$$
\varphi\left(x_{n}\right) \leq \gamma_{n}+\sum_{m=\alpha}^{n-1} f_{m} \phi\left(x_{m}\right)+\sum_{m=\alpha}^{n-1} g_{m} \psi\left(x_{m}\right), n \geq \alpha,
$$

where $\alpha$ is an integer, $\left\{f_{n}\right\}_{n=\alpha}^{\infty},\left\{g_{n}\right\}_{n=\alpha}^{\infty},\left\{\gamma_{n}\right\}_{n=\alpha}^{\infty}$ are nonnegative sequences, and $\left\{\gamma_{n}\right\}_{n=\alpha}^{\infty}$ is nondecreasing.

There are many reasons for studying (2). As an example, let $\left\{x_{n}\right\}_{n=0}^{\infty}$ be the population of a species in time periods $n=0,1, \ldots$. Let $\varphi$ measures the intellectual ability of the species and $\psi$ measures the living standard. It is reasonable that the increase in intellectual ability in two consecutive time periods, that is $\varphi\left(x_{n+1}\right)-\varphi\left(x_{n}\right)$, is proportional to $\varphi\left(x_{n}\right)$ and also to $\psi\left(x_{n}\right)$. Let $\varepsilon_{n}$ stands for nonnegative extrinsic factors such as knowledge from an outside civilization, then assuming a superposition effect, we then have

$$
\varphi\left(x_{n+1}\right)-\varphi\left(x_{n}\right)=f \varphi\left(x_{n}\right)+g \psi\left(x_{n}\right)+\varepsilon_{n}, n=0,1,2, \ldots,
$$

where $f$ and $g$ are positive porportionality constants. Summing the above equation, we then obtain

$$
\varphi\left(x_{n}\right)=\sum_{m=0}^{n-1} f \varphi\left(x_{m}\right)+\sum_{m=0}^{n-1} g \psi\left(x_{m}\right)+\gamma_{n},
$$

Received October 31, 2001; revised November 28, 2001.

2000 Mathematics Subject Classification. 39B05, 39A05.

Key words and phrases. Ou-Iang inequality, functional inequality. 
where $\gamma_{n}=\sum_{m=0}^{n-1} \varepsilon_{m}$.

In case $\phi$ is trivial and $\varphi$ is the identity function, the following two results are known (see e.g. [2, Theorem 15.7] and [2, Lemma 15.6]).

Lemma 1. Suppose $\phi$ is trivial and $\varphi$ is the identity function. Suppose $\left\{\gamma_{n}\right\}_{n=\alpha}^{\infty}$ is a positive sequence, and $\psi$ is a positive, nondecreasing and continuous function defined on $(0, \infty)$. Let

$$
G(u)=\int_{u_{0}}^{u} \frac{d t}{\psi(t)}, u, u_{0}>0 .
$$

If $\left\{x_{n}\right\}_{n=\alpha}^{\infty}$ is a nonnegative solution of (2), then

$$
x_{n} \leq G^{-1}\left(G\left(\gamma_{n}\right)+\sum_{m=\alpha}^{n-1} g_{m}\right)
$$

for $n=\alpha, \alpha+1, \ldots, \beta$, provided that

$$
G\left(\gamma_{n}\right)+\sum_{m=\alpha}^{n-1} g_{m} \in G(0, \infty), n=\alpha, \alpha+1, \ldots, \beta .
$$

We remark that when $n=\alpha$, the sum appearing in the above result is taken to be zero. This practice is in line with the general convention that empty sums are taken to be zero, and empty products to be one.

Lemma 2. Suppose $\phi$ is trivial and $\varphi$ is the identity function. Suppose further that $\gamma_{n}=\gamma+\sum_{m=\alpha}^{n-1} \sigma_{m}$ for $n \geq \alpha$, where $\left\{\sigma_{m}\right\}_{m=\alpha}^{\infty}$ is a nonnegative sequence, and $\psi$ is a positive, nondecreasing and continuous function defined on $[0, \infty)$. Let

$$
G(u)=\int_{u_{0}}^{u} \frac{d t}{\psi(t)}, u, u_{0}>0 .
$$

If $\left\{x_{n}\right\}_{n=\alpha}^{\infty}$ is a nonnegative solution of (2), then

$$
x_{n} \leq G^{-1}\left(G\left(\gamma_{n}\right)+\sum_{m=\alpha}^{n-1} g_{m}\right)
$$

for $n=\alpha, \alpha+1, \ldots, \beta$, provided that

$$
G\left(\gamma_{n}\right)+\sum_{m=\alpha}^{n-1} g_{m} \in G(0, \infty), n=\alpha, \alpha+1, \ldots, \beta .
$$

In case $\phi$ is the identity function, the following is known (see e.g. [2, Theorem 15.10]).

Lemma 3. Suppose $\phi$ and $\varphi$ are the identity function, and suppose $\psi$ is a nondecreasing and continuous function on $[0, \infty)$ such that $\psi(u)>0$ for $u>0$. Let

$$
G(x)=\int_{\beta}^{x} \frac{d t}{\psi(t)}, \beta, x>0
$$


and

$$
A_{n}=\gamma_{n}+\sum_{m=\alpha}^{n-1} \gamma_{m} f_{m} \prod_{i=m+1}^{n-1}\left(1+f_{i}\right), n \geq \alpha
$$

If $\left\{x_{n}\right\}_{n=\alpha}^{\infty}$ is a nonnegative solution of $(2)$, then

$$
x_{n} \leq G^{-1}\left(G\left(A_{n}\right)+\sum_{m=\alpha}^{n-1} g_{m} \prod_{i=m+1}^{n-1}\left(1+f_{i}\right)\right)
$$

for $n=\alpha, \alpha+1, \ldots, \beta$, provided

$$
G\left(A_{n}\right)+\sum_{m=\alpha}^{n-1} g_{m} \prod_{i=m+1}^{n-1}\left(1+f_{i}\right) \in G(0, \infty), n=\alpha, \alpha+1, \ldots, \beta
$$

In case $\varphi=\phi$, we may show the following.

Theorem 1. Suppose $\varphi=\phi$, and $\phi, \psi$ are continuous and nonnegative functions defined on $[0, \infty), \phi$ is increasing and $\psi$ is nondecreasing. Let

$$
\Omega(r)=\int_{\beta}^{r} \frac{d t}{\psi\left(\phi^{-1}(t)\right)}, r, \beta>0
$$

and

$$
\Gamma_{n}=\gamma_{n}+\sum_{m=\alpha}^{n-1} \gamma_{m} f_{m} \prod_{j=m+1}^{n-1}\left(1+f_{j}\right), n \geq \alpha
$$

If $\left\{x_{n}\right\}_{n=\alpha}^{\infty}$ is a nonnegative solution of (2), then

$$
x_{n} \leq \phi^{-1}\left(\Omega^{-1}\left[\Omega\left(\Gamma_{n}\right)+\sum_{m=\alpha}^{n-1} g_{m} \prod_{i=m+1}^{n-1}\left(1+f_{i}\right)\right]\right)
$$

for $n=\alpha, \alpha+1, \ldots, \beta$, provided

$$
\Omega\left(\Gamma_{n}\right)+\sum_{m=\alpha}^{n-1} g_{m} \prod_{i=m+1}^{n-1}\left(1+f_{i}\right) \in \Omega(0, \infty), n=\alpha, \alpha+1, \ldots, \beta
$$

Proof. Let

$$
u_{n}=\gamma_{n}+\sum_{m=\alpha}^{n-1} f_{m} \phi\left(x_{m}\right)+\sum_{m=\alpha}^{n-1} g_{m} \psi\left(x_{m}\right), n \geq \alpha
$$

Then $\left\{u_{n}\right\}_{n=\alpha}^{\infty}$ is nonnegative, $u_{\alpha}=\gamma_{\alpha}$ and

$$
\Delta u_{n}=\Delta \gamma_{n}+f_{n} \phi\left(x_{n}\right)+g_{n} \psi\left(x_{n}\right) \geq 0, n \geq \alpha .
$$


Since $\phi$ is increasing, thus $x_{n} \leq \phi^{-1}\left(u_{n}\right)$ for $n \geq \alpha$. As a consequence,

$$
\Delta u_{n} \leq \Delta \gamma_{n}+f_{n} u_{n}+g_{n} \psi\left(\phi^{-1}\left(u_{n}\right)\right), n \geq \alpha,
$$

which implies

$$
u_{n} \leq \gamma_{n}+\sum_{m=\alpha}^{n-1} f_{m} u_{m}+\sum_{m=\alpha}^{n-1} g_{m} \psi\left(\phi^{-1}\left(u_{n}\right)\right), n \geq \alpha .
$$

According to Lemma 3,

$$
u_{n} \leq \Omega^{-1}\left[\Omega\left(\Gamma_{n}\right)+\sum_{m=\alpha}^{n-1} g_{m} \prod_{i=m+1}^{n-1}\left(1+f_{i}\right)\right]
$$

for $n=\alpha, \alpha+1, \ldots, \beta$, provided that

$$
\Omega\left(\Gamma_{n}\right)+\sum_{m=\alpha}^{n-1} g_{m} \prod_{i=m+1}^{n-1}\left(1+f_{i}\right) \in \Omega(0, \infty), n=\alpha, \alpha+1, \ldots, \beta .
$$

Finally, (5) follows from (6) and $\phi\left(x_{n}\right) \leq u_{n}$. The proof is complete.

As a corollary, let $\phi(u)=u^{p}$ and $\psi(u)=u^{q}$ where $p, q$ are distinct positive numbers. The function $\Omega$ defined by (4) is then equal to

$$
\Omega(r)=\int_{1}^{r} \frac{d t}{t^{q / p}}=\frac{p}{p-q}\left(r^{(p-q) / p}-1\right), r>0,
$$

and

$$
\Omega^{-1}(t)=\left(\frac{p-q}{p} t+1\right)^{p /(p-q)}
$$

where

$$
\Omega(0, \infty)=\operatorname{dom} \Omega^{-1}=\left\{\begin{array}{ll}
(p /(q-p), \infty) & p>q \\
(-\infty, p /(q-p)) & p<q
\end{array} .\right.
$$

Therefore, the conclusion of Theorem 1 is now

$$
x_{n} \leq\left\{\Gamma_{n}^{(p-q) / p}+\frac{p-q}{p} \sum_{m=\alpha}^{n-1} g_{m} \prod_{i=m+1}^{n-1}\left(1+f_{i}\right)\right\}^{1 /(p-q)}
$$

for $n=\alpha, \alpha+1, \ldots, \beta$, provided that

$$
\Gamma_{n}^{(p-q) / p}+\frac{p-q}{p} \sum_{m=\alpha}^{n-1} g_{m} \prod_{i=m+1}^{n-1}\left(1+f_{i}\right) \in \operatorname{dom} \Omega^{-1}, n=\alpha, \alpha+1, \ldots, \beta .
$$

We remark that the above conclusion is different from the result stated in $[1$, Theorem 1] for equation (1), and therefore Theorem 1 is not a direct generalization but a complementary result of Theorem 1 in [1]. 
Theorem 2. Suppose $\varphi$ is the identity function. Suppose $\gamma_{\alpha}>0$. Suppose further that $\phi, \psi$ are positive, continuous and nondecreasing on $[0, \infty)$. Let

$$
\begin{gathered}
\Psi(u)=\int_{u_{0}}^{u} \frac{d t}{\psi(t)}, u, u_{0}>0, \\
\Phi(u)=\int_{u_{0}}^{u} \frac{d t}{\phi(t)}, u, u_{0}>0,
\end{gathered}
$$

and $\Phi(u+v) \geq \Phi(u)+\Phi(v)$ for $u, v>0$, and $\lim _{u \rightarrow+\infty} \Phi(u)=+\infty$. If $\left\{x_{n}\right\}_{n=\alpha}^{\infty}$ is a nonnegative solution of (2), then

$$
x_{n} \leq \Psi^{-1}\left(\Psi\left(\gamma_{n}+\Phi^{-1}\left(\sum_{m=\alpha}^{n-1} f_{m}\right)\right)+\sum_{m=\alpha}^{n-1} g_{m}\right)
$$

for $n=\alpha, \alpha+1, \ldots, \beta$, provided that

$$
\Psi\left(\gamma_{n}+\Phi^{-1}\left(\sum_{m=\alpha}^{n-1} f_{m}\right)\right)+\sum_{m=\alpha}^{n-1} g_{m} \in \Psi(0, \infty), n=\alpha, \alpha+1, \ldots, \beta .
$$

Proof. Let

$$
u_{n}=\gamma_{n}+\sum_{m=\alpha}^{n-1} f_{m} \phi\left(x_{m}\right)+\sum_{m=\alpha}^{n-1} g_{m} \psi\left(x_{m}\right), n \geq \alpha .
$$

Then $\left\{u_{n}\right\}_{n=\alpha}^{\infty}$ is a nonnegative sequence, $u_{\alpha}=\gamma_{\alpha}$ and

$$
\Delta u_{n}=\Delta \gamma_{n}+f_{n} \phi\left(x_{n}\right)+g_{n} \psi\left(x_{n}\right) \geq 0, n \geq \alpha .
$$

Since $x_{n} \leq u_{n}$ for $n \geq \alpha$,

$$
\Delta u_{n} \leq \Delta \gamma_{n}+f_{n} \phi\left(u_{n}\right)+g_{n} \psi\left(u_{n}\right), n \geq \alpha,
$$

which implies

$$
u_{n} \leq\left(\gamma_{\alpha}+\sum_{m=\alpha}^{n-1}\left(g_{m} \psi\left(u_{n}\right)+\Delta \gamma_{m}\right)\right)+\sum_{m=\alpha}^{n-1} f_{m} \phi\left(u_{m}\right), n \geq \alpha .
$$

In view of Lemma 2 ,

$$
u_{n} \leq \Phi^{-1}\left(\Phi\left(\gamma_{\alpha}+\sum_{m=\alpha}^{n-1}\left(g_{m} \psi\left(u_{m}\right)+\Delta \gamma_{m}\right)\right)+\sum_{m=\alpha}^{n-1} f_{m}\right)
$$

for $n=\alpha, \alpha+1, \ldots, \beta$, provided

$$
\Phi\left(\gamma_{\alpha}+\sum_{m=\alpha}^{n-1}\left(g_{m} \psi\left(u_{m}\right)+\Delta \gamma_{m}\right)\right)+\sum_{m=\alpha}^{n-1} f_{m} \in \Phi(0, \infty), n=\alpha, \alpha+1, \ldots, \beta .
$$


Since $\lim _{u \rightarrow \infty} \Phi(u)=+\infty$, the last condition holds for any $\beta$. Thus (7) holds for all $n \geq \alpha$. Next, note that the conditions imposed on $\Phi$ imply $\Phi^{-1}(u+v) \leq \Phi^{-1}(u)+\Phi^{-1}(v)$ for all $u, v \in \Phi(0, \infty)$. Thus,

$$
\begin{aligned}
u_{n} & \leq \gamma_{\alpha}+\sum_{m=\alpha}^{n-1}\left(g_{m} \psi\left(u_{m}\right)+\Delta \gamma_{m}\right)+\Phi^{-1}\left(\sum_{m=\alpha}^{n-1} f_{m}\right) \\
& =\left(\gamma_{n}+\Phi^{-1}\left(\sum_{m=\alpha}^{n-1} f_{m}\right)\right)+\sum_{m=\alpha}^{n-1} g_{m} \psi\left(u_{m}\right) .
\end{aligned}
$$

Since $\left\{\gamma_{n}+\Phi^{-1}\left(\sum_{m=\alpha}^{n-1} f_{m}\right)\right\}$ is a positive and nondecreasing sequence, according to Lemma 1,

$$
x_{n} \leq u_{n} \leq \Psi^{-1}\left(\Psi\left(\gamma_{n}+\Phi^{-1}\left(\sum_{m=\alpha}^{n-1} f_{m}\right)\right)+\sum_{m=\alpha}^{n-1} g_{m}\right)
$$

for $n=\alpha, \alpha+1, \ldots, \beta$, provided

$$
\Psi\left(\gamma_{n}+\Phi^{-1}\left(\sum_{m=\alpha}^{n-1} f_{m}\right)\right)+\sum_{m=\alpha}^{n-1} g_{m} \in \Psi(0, \infty), n=\alpha, \alpha+1, \ldots, \beta .
$$

The proof is complete.

As an application, consider the functional relation

$$
x_{n} \leq\left(a_{n}+\sum_{m=\alpha}^{n-1} F_{m} w\left(x_{m}\right)\right)\left(b_{n}+\sum_{m=\alpha}^{n-1} G_{m} w\left(x_{m}\right)\right), n \geq \alpha,
$$

where $\left\{F_{n}\right\}_{n=\alpha}^{\infty},\left\{G_{n}\right\}_{n=\alpha}^{\infty},\left\{a_{n}\right\}_{n=\alpha}^{\infty},\left\{b_{n}\right\}_{n=\alpha}^{\infty},\left\{\Delta a_{n}\right\}_{n=\alpha}^{\infty}$ and $\left\{\Delta b_{n}\right\}_{n=\alpha}^{\infty}$ are nonnegative sequences and $w$ is a positive, nondecreasing and continuous function defined on $[0, \infty)$, and $\alpha_{\alpha} b_{\alpha}>0$. Denote the right hand side of (8) by $z_{n}$. Suppose $\left\{x_{n}\right\}_{n=\alpha}^{\infty}$ is a nonnegative solution of $(8)$. Then

$$
\begin{aligned}
\Delta z_{n}= & \left(a_{n}+\sum_{m=\alpha}^{n-1} F_{m} w\left(x_{m}\right)\right)\left(\Delta b_{n}+G_{n} w\left(x_{n}\right)\right) \\
& +\left(\Delta a_{n}+F_{n} w\left(x_{n}\right)\right)\left(b_{n+1}+\sum_{m=\alpha}^{n} G_{m} w\left(x_{m}\right)\right) \\
= & a_{n} \Delta b_{n}+b_{n+1} \Delta a_{n}+\left(a_{n} G_{n}+b_{n+1} F_{n}\right) w\left(x_{n}\right) \\
& +\Delta b_{n} \sum_{m=\alpha}^{n-1} F_{m} w\left(x_{m}\right)+\Delta a_{n} \sum_{m=\alpha}^{n} G_{m} w\left(x_{m}\right) \\
& +G_{n} w\left(x_{n}\right) \sum_{m=\alpha}^{n-1} F_{m} w\left(x_{m}\right)+F_{n} w\left(x_{n}\right) \sum_{m=\alpha}^{n} G_{m} w\left(x_{m}\right) \\
\geq & 0
\end{aligned}
$$


for $n \geq \alpha$. If we assume further that $w$ is nondecreasing, then

$$
\begin{aligned}
\Delta z_{n} \leq & \Delta\left(a_{n} b_{n}\right)+\left(a_{n} G_{n}+b_{n+1} F_{n}\right) w\left(z_{n}\right) \\
& +\left(\Delta b_{n} \sum_{m=\alpha}^{n-1} F_{m}+\Delta a_{n} \sum_{m=\alpha}^{n} G_{m}\right) w\left(z_{n}\right) \\
& +\left(G_{n} \sum_{m=\alpha}^{n-1} F_{m}+F_{n} \sum_{m=\alpha}^{n} G_{m}\right) w^{2}\left(z_{n}\right) .
\end{aligned}
$$

Let

and

$$
\begin{gathered}
f_{n}=a_{n} G_{n}+b_{n+1} F_{n}+\Delta b_{n} \sum_{m=\alpha}^{n-1} F_{m}+\Delta a_{n} \sum_{m=\alpha}^{n} G_{m}, n \geq \alpha, \\
g_{n}=G_{n} \sum_{m=\alpha}^{n-1} F_{m}+F_{n} \sum_{m=\alpha}^{n} G_{m}, n \geq \alpha, \\
W_{1}(u)=\int_{u_{0}}^{u} \frac{d t}{w(t)}, u, u_{0}>0,
\end{gathered}
$$

$$
W_{2}(u)=\int_{u_{0}}^{u} \frac{d t}{w^{2}(t)}, u, u_{0}>0 .
$$

According to Theorem 2, if we make the additional assumption that $W_{1}$ satisfies $W_{1}(u+$ $v) \geq W_{1}(u)+W_{1}(v)$ for $u, v>0$, and $\lim _{u \rightarrow+\infty} W(u)=+\infty$, then

$$
x_{n} \leq W_{2}^{-1}\left(W_{2}\left(a_{n} b_{n}+W_{1}^{-1}\left(\sum_{m=\alpha}^{n-1} f_{n}\right)\right)+\sum_{m=\alpha}^{n-1} g_{m}\right)
$$

for $n=\alpha, \alpha+1, \ldots, \beta$, provided that

$$
W_{2}\left(a_{n} b_{n}+W_{1}^{-1}\left(\sum_{m=\alpha}^{n-1} f_{n}\right)\right)+\sum_{m=\alpha}^{n-1} g_{m} \in W_{2}(0, \infty), n=\alpha, \alpha+1, \ldots, \beta .
$$

As another example, we consider our equation (3). It is reasonable to assume that $\phi, \psi$ are continuous and nonnegative functions defiend on $[0, \infty), \phi$ is increasing and $\psi$ is nondecreasing. For simplicity, we will also assume that $\varepsilon_{n}=\varepsilon>0$ for all $n$. Then letting

$$
\Omega(r)=\int_{\beta}^{r} \frac{d t}{\psi\left(\phi^{-1}(t)\right)}, r, \beta>0,
$$

and

$$
\begin{aligned}
\Gamma_{n} & =\gamma_{n}+\sum_{m=\alpha}^{n-1} \gamma_{m} f_{m} \prod_{j=m+1}^{n-1}\left(1+f_{j}\right) \\
& =n \varepsilon+m f \varepsilon \sum_{m=0}^{n-1}(1+f)^{n-m-1} \\
& =n \varepsilon+m \varepsilon\left((1+f)^{n}-1\right),
\end{aligned}
$$


if $\left\{x_{n}\right\}_{n=\alpha}^{\infty}$ is a nonnegative solution of (2), then

$$
\begin{aligned}
x_{n} & \leq \phi^{-1}\left(\Omega^{-1}\left[\Omega\left(\Gamma_{n}\right)+g \sum_{m=0}^{n-1}(1+f)^{n-m-1}\right]\right) \\
& =\phi^{-1}\left(\Omega^{-1}\left[\Omega\left(\Gamma_{n}\right)+g \frac{(1+f)^{n}-1}{f}\right]\right)
\end{aligned}
$$

for $n=\alpha, \alpha+1, \ldots, \beta$, provided

$$
\Omega\left(\Gamma_{n}\right)+g \frac{(1+f)^{n}-1}{f} \in \Omega(0, \infty), n=\alpha, \alpha+1, \ldots, \beta .
$$

\section{References}

[1] W. R. Li and S. S. Cheng, Discrete Ou-Iang type inequalities, Tamkang J. Math. 31(2000), 305-309.

[2] D. Bainov and P. Simeonov, Integral Inequalities and Applications, Kluwer Academic Publishers, 1992.

Department of Mathematics, Binzhou Normal College, Binzhou, Shandong 256604, P. R. China.

Department of Mathematics, Tsing Hua University, Hsinchu, Taiwan 30043, R. O. C. 